\title{
A STRUCTURED APPROACH FOR SYNCHRONISING THE APPLICATIONS OF FAILURE MODE AND EFFECTS ANALYSIS
}

\author{
Mansoor ALRUQI \\ University of Nottingham \\ Shaqra University \\ Martin BAUMERS, David BRANSON III \\ University of Nottingham \\ Robert FARNDON \\ Rolls-Royce plc
}

\begin{abstract}
:
Failure Mode and Effects Analysis (FMEA) is a systematic approach for evaluating the potential failure modes in a system, and is mainly employed in three distinct tasks labelled: (1) Functional FMEA - evaluating those failures associated with product functional definition, (2) Design FMEA - analysing those failures associated with design definition and (3) Process FMEA - assessing potential failures in manufacturing and assembly processes. The literature review has shown limited works on the field of synchronising these different tasks into a working model. To address this gap, this research developed a framework for integrating these tasks of FMEAs, and then qualitatively validating the proposed framework. This research adopted a semi-structured questionnaire to collect experts' feedback and validate the proposed framework. The t-test was then employed to evaluate the collected feedback. The findings highlight that the proposed framework is applicable and could facilitate the synchronisation of the different tasks of FMEA. This research presents a methodological approach for executing and synchronising FMEAs. Therefore, the proposed framework is practically relevant as an aid for the practitioners in catching the cascading failures and reducing the relevant impact.
\end{abstract}

Key words: Axiomatic Design, Design Structure Matrix, FMEA, synchronicity

\section{INTRODUCTION}

The investigation of failures and their prevention throughout a design process forms an effective approach for reducing the number of failures in manufacturing, as failures could be prevented and minimised at an early stage and in a proactive manner [1, 2, 3, 4]. To address this issue, Failure Mode and Effects Analysis (FMEA) was developed to proactively evaluate the potential failures in a product, process or service, as well as identifying actions to reduce and mitigate risks. The associated benefits of implementing FMEA likely originate from its ability to recognise and mitigate failures in advance, making them less costly to address $[5,6]$. FMEA has always been an important factor and a key dimension in improving the performance of the developmental process of products, as well as being able to generate lessons that can be learned for proactive management $[2,7,8]$.

To achieve a high quality, FMEA is executed through six main moves as demonstrated in Figure 1 [2]. Starting from the FMEA scope, its deployment should be limited to specific systems and components; so that is for establishing the boundaries and maximising the benefits of its deployment [2]. Then, the functions concerned and the associated failure modes should be determined to understand the impact of those modes and assess the possible improvement actions [2]. The next is to perform a detailed analysis and prioritise the criticality of failures [2]. Lastly, the FMEA review is the activity of evaluating the FMEA conducted [2]. Every FMEA process should have an owner, who can revise the progress of FMEA and approve the relevant outputs [2]. 


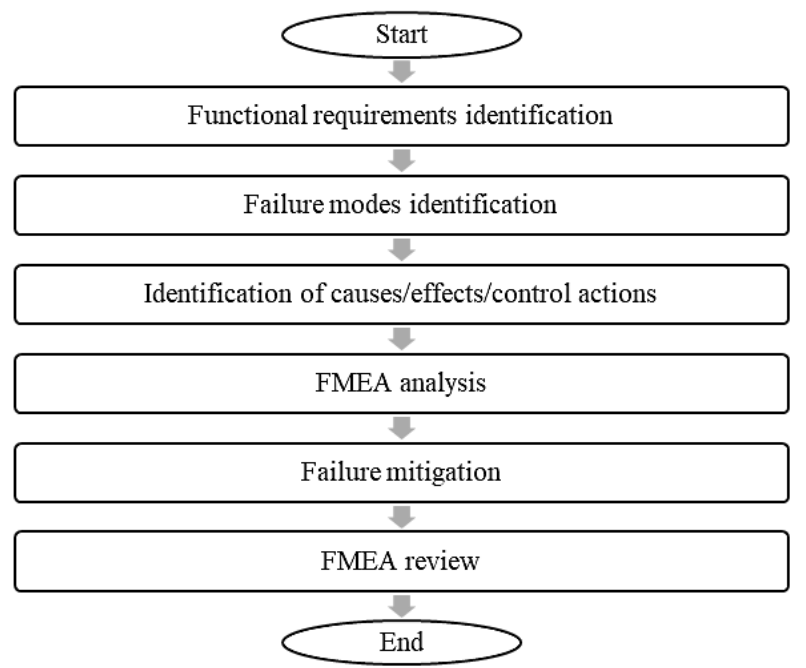

Fig. 1 The processes for executing FMEA

As a core element in the reliability and quality fields of product development, FMEA is adopted in three distinct applications [2, 9]: (1) Functional FMEA - evaluating product and system functions-related failure modes, (2) Design FMEA - appraising the failure modes associated with sub-system functions and components designs and (3) Process FMEA - assessing failures identified in manufacturing and assembly processes. To maximise the benefits of applying FMEA, these different tasks of FMEA must be synchronised as presented in Figure 2 [1, 2, 3, 4, 5, 10].

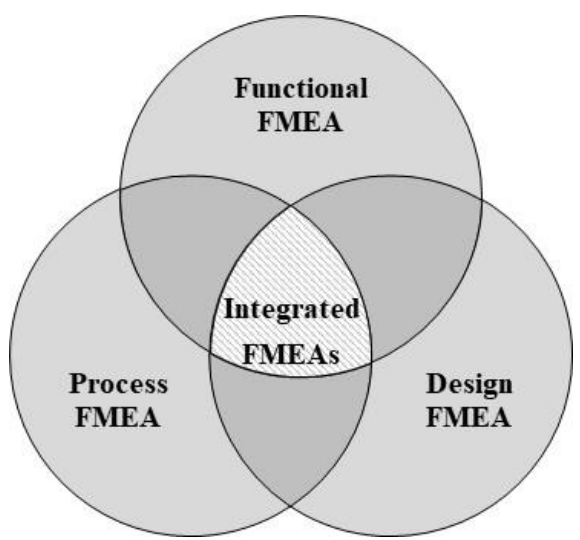

Fig. 2 Synchronising FMEA applications

Researching the requirements of interlinking FMEAs is essential for a couple of purposes. First, FMEAs interlinked within complex applications are required to transform functional requirements into the process domain [3]. Second, the interlinkage between FMEA applications can effectively identify and capture the cascading failures, those failures escape between Functional, Design and Process FMEAs $[1,2,3,4,5,10]$. Third, it allows one to look at failures from both generic and downstream perspectives [2, 3]. Finally, the interlinking of FMEAs could provide orientation to ensure the failures identified are traceable and thus, making and correlating the appropriate control actions for the related causes [3].

FMEA deployment, however, has been determined as a time-consuming methodology due to the resources required for its execution [11, 12]. Because the current form of FMEA in its own application is not able to identify the primary functional requirements and the associated failure modes $[4,10,11,13]$. This means that it is difficult for the current form of FMEA to capture these requirements in a detailed bottom-up approach, which in turn, it impedes the systematic transfer of these requirements to the process domain, leading to an inability to shift and trace the product requirements and catch the associated failures that cascade over Functional, Design and Process FMEAs $[4,10,13]$. This can be attributed to the level of complexity and depth of analysis required to perform FMEA $[2,3,11]$.

Functional, Design and Process FMEAs are usually carried out separately, and so, as a result, several links in between are generally not understood, which forms a challenge for the full and effective execution of FMEAs [2, 3, 14]. Carlson [2] states that all FMEAs are dependent upon each other, and every individual FMEA application is used as a form of orientation and guidance for the other. For example, Design FMEA outputs are used to generate Process FMEA [2]. Thus, synchronising all FMEA procedures into one practice can lead to efficiency in their applications in terms of a more optimal resource usage [2, 3, 14]. Although, there is no clear definition for the synchronicity between FMEAs, it can be described as the process of integrating all features of Functional, Design and Process FMEAs where failure modes can be captured at any form of FMEA and their effects can be cascaded over to different modes of FMEA [2]. As a result, this could improve the operations of deploying FMEA over different production levels and maximise the positive impacts of its deployment on either the users or the system/process is under examination $[2,3]$.

The remainder of the paper is structured as follows. The next section discusses the literature on the current works devoted to improving the synchronicity of FMEAs. The following section outlines the methodologies suggested for developing the framework as well as the research method applied for evaluating the developed framework including the statistical inference model of the t-test. The subsequent section presents the research' results and discusses the limitations. The paper finally concludes with a summary and brief suggestions for future work.

\section{LITERATURE REVIEW}

Few studies have considered this strategic element by presenting such improvements. Gu, Cheng and Qiu [15], Shaker, Shahin and Jahanyan [16] and Hassan et al. [17] presented an improved FMEA model that aims to synchronise both Functional and Design FMEAs. Their model was based on the combination of Quality Functional Deployment (QFD), a design management tool that transforms the customer needs into engineering characteristics. Kmenta and Ishii [4] suggested the Scenario-Based FMEA approach that is organised around the failure chain (i.e. another meaning of the cascading failure) to track the effects of failures over the product life cycle, from the failure root causes across the intermediate effects to end effects. This approach is potentially applicable to connect Functional with Design FMEAs; chasing the failure effects 
down-up to the system level, vice versa for chasing failure causes up-down to component level [1]. Also, this approach could be used to interrelate Design with Process FMEAs through transferring and tracing design-related issues over Process FMEA. However, this framework could be improved if the failure scenario is represented through a schematic approach to correlate these concerns to the associated manufacturing and assembly processes [3].

In addition to the Scenario-Based FMEA approach, Cooper [18] developed and used a systematised approach for decomposing system functions and capturing all critical failure modes into Design FMEA; just using P-Diagram to trace the next level effects, as a way for catching the cascading failures. P-Diagram, sometimes called Parameter Diagram, is a tool used to show the system boundaries including the input resources, noise factors and targeted outcomes. Cooper's [18] work has only focused on the preparation process for deploying Design FMEA by using the decomposition table; however, this work has failed to acknowledge the significance of the linkage between all FMEAs. To improve this work, it would be more effective if this approach is supported with a structured tree to represent the system hierarchy. Having this tree developed could help to trace and link system levels and parallel to the process domain, and consequently catch the cascading failures $[3,19]$.

A sound FMEA interlinkage, as proposed by Teng et al. [3] and Mohsen and Cekecek [19], involves creating a taxonomy for a particular system to scope FMEA analysis and reduce the complexity of the given design. As suggested by Suh [20], this can be provided by Axiomatic Design method ( $A D)$. AD is a design management methodology that systematically transforms customer needs and functional requirements into design and process domains. Underpinned by the decomposition tree and zigzagging, $A D$ implementation plays an important role in controlling the decomposition and transformation of functional requirements into the design and process domains at each level and between each domain $[19,20]$. Well-developed $A D$ generates an aggregated hierarchy of the functional, design and process domains, which addresses a good overview of the product structure, as well as accessible learning and understanding $[19,20,21]$. This overview is required for evaluating complex systems coupled with quantities of information, such as in the case of aeroengines, which could effectively lead to tracing the cascading failures and, most importantly, identifying their root causes [22]. The hierarchy combined these domains could provide an additional indication to ensure functional requirements are traceable to a certain level, that would show that the system design and process in question is fully determined and controlled [20, 21].

Arcidiacono and Campatelli [23] developed a framework combining AD and Fault Tree Analysis (FTA) methodologies with FMEA to develop a reliability tree that could promote the success of FMEA implementation. FTA is a topdown risks assessment approach used to determine and quantify the likelihood of failure occurrence. This work has shown that when FTA and AD methods combined with
FMEA, a 50\% saving in resources utilised in implementing FMEA in the complex system was achieved. However, this work is limited to Functional and Design FMEA, which has failed to address the linkage to Process FMEA.

Although these approaches outlined above are useful in terms of synchronising FMEAs, they are still limited by their scope to either only linking Functional with Design FMEAs or Design with Process FMEAs, which ignores the importance of the full link between FMEA applications. To the best of the authors' knowledge, the extant literature has demonstrated a dearth of works devoted to synchronising Functional, Design and Process FMEAs and adapt the application of AD method to integrate all of these different tasks of FMEA. This knowledge gap is critical to an important quality and reliability tool like FMEA. As such, the community of practice would require a guide on how to link the Functional, Design and Process FMEAs. Therefore, this article aims to investigate the requirements to link these applications by developing a conceptualised framework that involves the co-adoption of $A D$ method. To evaluate the developed framework, this work has been further supported with a complementary evaluation to assess the level of acceptance through the feedback collected from the field-related individuals, which were then fed into a t-test model for analysis.

Our contribution is twofold: first, we advance the literature on FMEA field by proposing a hybrid risk assessment approach that involves synchronising Functional, Design and Process FMEAs. This approach can offer a structured method for systemically identifying the cascading failures, causes and the potential effects across the application of Functional, Design and Process FMEAs. This work constitutes a relatively new area, which has emerged due to the lack of research in the current literature on integrating Functional, Design and Process FMEAs and because of the knowledge gap in utilising $A D$ method to synchronise FMEA applications. This work highlights the compatibility between $A D$ and FMEA methods, as well as the relevant ability to streamline the operations for implementing FMEA. We believe that this framework would guide the practitioners in efficiently deploying FMEA in their practices, thereby reducing the associated cost. Second, we provide a new perspective on interlinking the product development domains, which has the potential to contribute towards minimising the manufacturing disruptions and downtime.

\section{METHODOLOGY}

There are three sections in this segment. The first section outlines the potential extension of utilising AD and Design Structure Matrix (DSM) approaches to integrate Functional, Design and Process FMEAs. DSM is suggested as a supportive approach to perform $A D$ and manage the coupling between elements of each phase in AD deployment. A novel framework for integrating Functional, Design and Process FMEAs is described in the following section. Finally, this segment presents the research method applied to evaluate the developed framework. 


\section{Axiomatic Design (AD)}

$A D$ method was developed to logically provide a foundation for the designers to improve the design workflows [20]. AD aims to reduce the lead time and the cost associated with the product development processes, by minimising the random search processes and allowing to catch customer needs at a detailed level [20]. It also helps to determine the best possible designs based on two axioms explained in the following section [20].

Four elements are involved in employing AD: domains, hierarchy, zigzagging and axioms. Domains are the operational hub for facilitating the definition of a product [20]. $A D$ involves four design domains operating as a logical succession [20]: (1) the customer domain, (2) the functional domain, (3) the physical domain and (4) the process domain.

As presented in Figure 3 these domains consist of four elements, namely: customer needs (CNs), functional requirements (FRs), design parameters (DPs) and process variables (PVs). CNs are the customers' desires to fulfil their needs. FRs are the functions identified to deliver the overall performance of a product. DPs are the physical characteristics that represent and implement FRs. PVs are the attributes of controlling and building FRs and DPs. Each domain defines the targets to achieve, and the next domain represents the solutions to accomplish these targets [20].

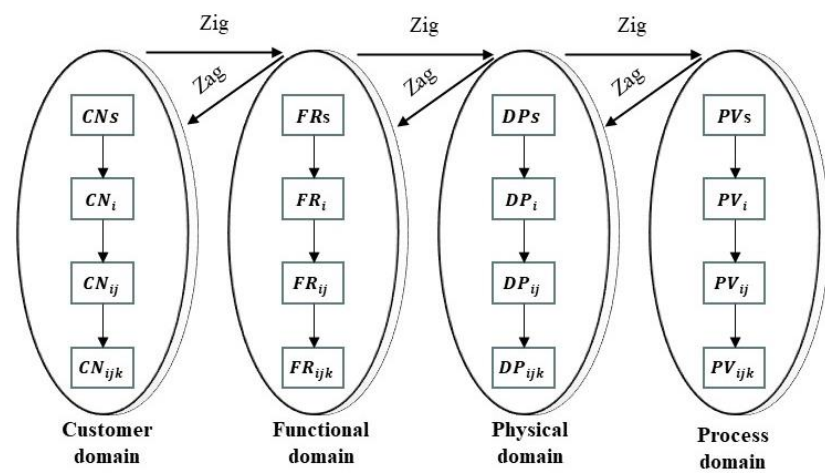

Fig. 3 AD domains, adapted from Suh

Source: [20].

Then, hierarchy is the taxonomic operational process for extending these domains to embody the system architecture. More so, zigzagging is the iterative process for vertically and horizontally decomposing these domains: where zig stands for determining the CNs/FRs/DPs/PVs and zag stands for locating the next level of FRs/DPs/PVs. At an increasing level of detail, these methodologies' elements provide an iterative structure of product functions that can be cascaded to their solutions [19, 20, 24, 25, 26, 27]. Finally, axioms are the set of rules defined to provide the boundaries and references for managing the design workflow and resulted in, potentially creating such robust design [20]. AD introduces two axioms: independence and information axioms. The former requires FRs to be independent and ensure that each FR does not affect the other FRs, while the latter requires the minimisation of design contents. These axioms are translated into various corollaries and theorems, which can be found in Suh [20].
To explain how AD works, the focus in this study is maintained on FRs-DPs-PVs domains. FRs are cascaded into DPs and PVs domains throughout iterator chains [20]. AD deployment process commences with setting FRs, DPs and PVs domains to define the abstraction level of the product development process, then establishing the hierarchy for each domain in parallel [20]. Having the first level been identified, the next step is to link these domains using the zigzagging process: a step for generating the system architecture and defining the next level of these domains $[24,25]$. The decomposition process is carried out until the final leaf of all branches of the established hierarchy has been reached, which reflects that the design under evaluation is understood and completed [20].

This hierarchical mapping of AD method is of advantage, by shifting the design process to be customer-oriented $[19,20]$. This means that the design is customer-driven and the associated process parameters are described to address the relative critical quality characteristics of design, as well as treating them in the first priority [19, 20]. This feature of $A D$ method also emphasises on the innovation of design and manufacturing process $[19,20]$.

Part of AD is Design Matrix (DM), which is employed with $m$ rows and $n$ columns to facilitate and mathematically illustrate the correlation between all domains demonstrated and ensure consistency among them [20]. DM should produce the following scenarios [20]: (1) coupled, (2) decoupled and (3) uncoupled designs. Decoupled and uncoupled scenarios are the only scenarios that can fulfil the requirement of the independence axiom [20]. For producing these required scenarios, DM must yield either diagonal or triangular matrixes [20]. Inspired from Suh [20], equations ( 1 ) and (2) below exhibit the simple illustrative diagonal and triangular matrixes: triangular matrix can be the opposite, where the upper triangular element only equals zero.

$$
\begin{aligned}
& {\left[\begin{array}{l}
F R_{1} \\
F R_{2}
\end{array}\right]=\left[\begin{array}{ll}
1 & 0 \\
0 & 1
\end{array}\right]\left[\begin{array}{l}
D P_{1} \\
D P_{2}
\end{array}\right]} \\
& {\left[\begin{array}{l}
F R_{1} \\
F R_{2}
\end{array}\right]=\left[\begin{array}{ll}
1 & 1 \\
0 & 1
\end{array}\right]\left[\begin{array}{l}
D P_{1} \\
D P_{2}
\end{array}\right]}
\end{aligned}
$$

Although AD application is argued to facilitate and ease the conceptual design process via the application of the independence and information axioms, therefore, reduce the complexity of the design [20], utilising these axioms in real applications are problematic due to the associated cost and complexity $[22,28]$. To support this argument, Frey et al. [22] concern that designing independent FRs at a detailed level is impossible for the application of aeroengine manufacturing particularly the modern design applications. For example, the modern jet engines present coupled designs at both system and component levels of the engine control system, and across design and manufacture of the component level of modern compressor, as observed in blisks [22]. However, partial fulfilment of these axioms can be considered as a feasible design model [22]. To address this imperfection of $A D$, Guenov and Barker [27] proposed an improvement approach by integrating AD method with Design Structure Matrix approach 
(DSM) to improve the applicability of AD and manage the coupled designs.

\section{Design Structure Matrix (DSM)}

DSM is a design matrix used to design and represent the interactive elements of the coupled designs $[29,30]$. It is a design management tool that is considered good for representing the interactions between design elements and improving the design process's efficiency [29, 30]. It is being recognised for its capability to decompose a system into constituent components, understand, model the associations between design elements and evaluate the possible re-integration of them [29].

In the basic DSM analysis, the primary goal is to minimise feedback loops and their scope by re-structuring or re-architecting the process, where the relevant square matrix is re-sequenced [29]. DSM has been used for such complex applications characterised by complex links of dependent components [29]. There are various applications of DSM, the common for design process is componentbased DSM, which is used to model the components/subsystem or system architecture.

DSM works first by listing the design elements in a square matrix and then marking the relation to each other. The next step is partitioning, reordering DSM's rows and columns in which DSM is transformed into lower triangular form; to potentially reduce the size of feedback loops [29]. The last step of DSM application is clustering the elements reordered based on common purposes, where to group the elements interconnected for further improvements and new insights into system decomposition and re-integration [29].

However, DSM has been recognised as less effective in the application of designing new products; as it is just being able to manage the already made designs [30]. Guenov and Barker [27] proposed using the integration of $A D$ and DSM methods for developing a new design, because $A D$ application is more useful for defining the design elements and presenting the link between domains [20], while DSM is a great strength for modelling the interactive design elements $[29,30]$. Jefferson, Ratchev and Crossley [26] have augmented the work of Guenov and Barker [27] to model a reconfigurable assembly process for aerospace applications.

\section{AD-DSM-FMEA Framework}

Figure 4 presents the theoretical framework of the integrated AD-DSM-FMEA.

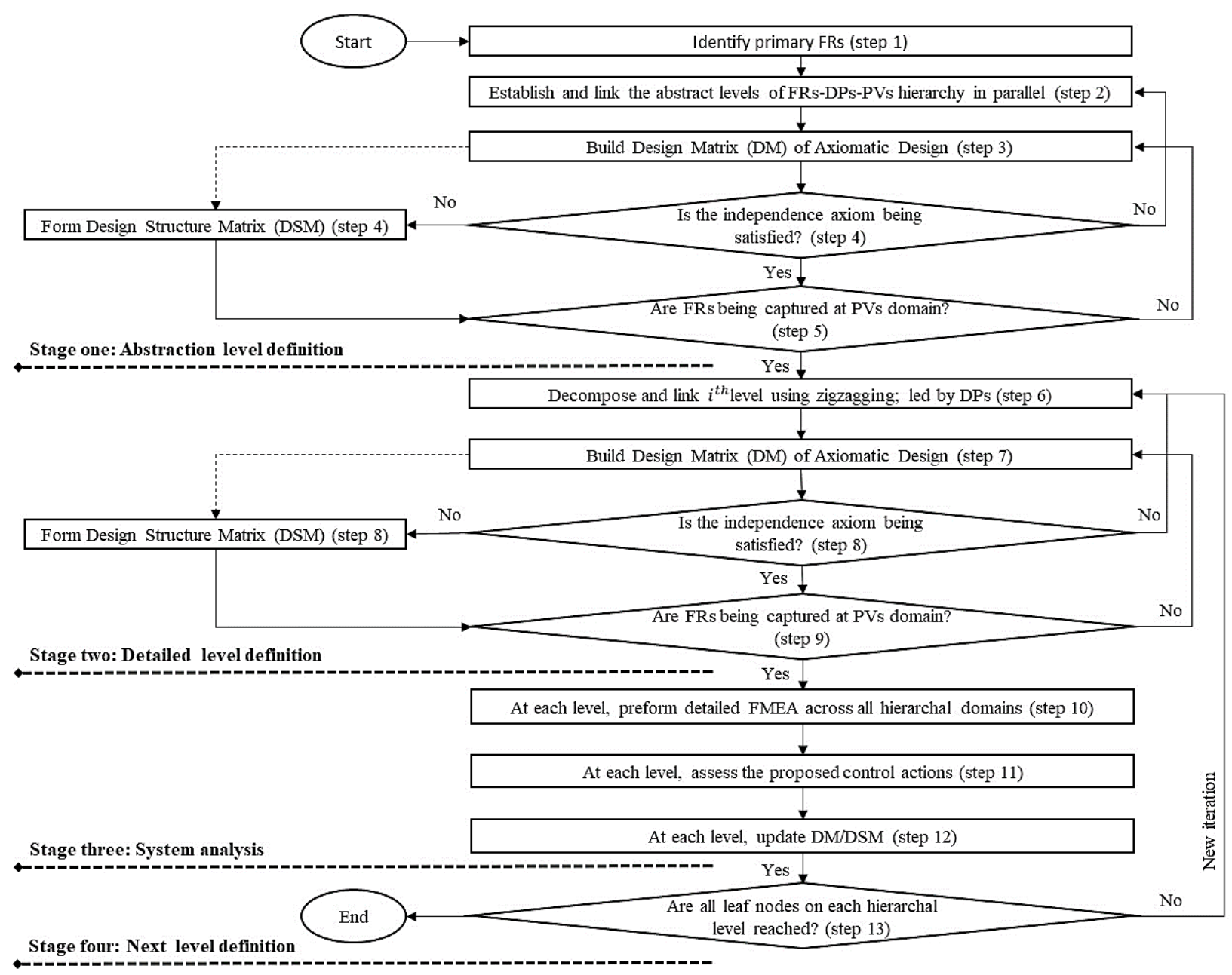

Fig. 4 The proposed conceptual framework of integrated AD-DSM-FMEA 
It comprises four stages, made of a set of loops, and organised to synchronise Functional, Design and Process FMEAs - thereby enabling the cascading failures to be captured. AD procedures are mainly adopted to manage the process of decomposing the system examined and to link FMEA applications. DSM approach is also combined to provide full coverage for managing the coupling issue between design elements. The term 'framework' has been used broadly in the engineering design domain within different contexts and meanings, as presented in Arcidiacono and Campatelli [23] and Pepe et al. [31].

The proposed framework is formed of 13 steps, which are grouped into four stages: abstraction level definition, detailed level definition, system analysis and next level definition. Each stage acts towards synchronising FMEAs, departing from defining the analysis scope of FMEA deployment and the introduction of the top level requirements of the product system and leading to establishing the related design features and process variables. Once these objectives are complete, the framework evaluates the associated failures, next if any, introducing and assessing the next system level. The following subsections explain each stage in detail.

\section{Stage one: Abstraction Level Definition}

This stage describes the process of identifying the scope of FMEA analysis and establishing the first level hierarchy of functional, design and process domains. It strives to answer the question "What must the system have to meet the customer needs?". To craft the AD hierarchy and set up the scope of FMEA analysis, the $1^{\text {st }}$ step identifies the primary functional requirements (FRs), design parameters (DPs) and process variables (PVs) of the system in question. The $2^{\text {nd }}$ step is to establish and link the abstraction level of functional, design and process domains in a parallel way to form the system taxonomy. Theoretically, this step horizontally decomposes FRs into DPs, then to the corresponding PVs. As illustrated in Figure 5, this step develops the overall picture and conceptualisation of the design and its embodiment.

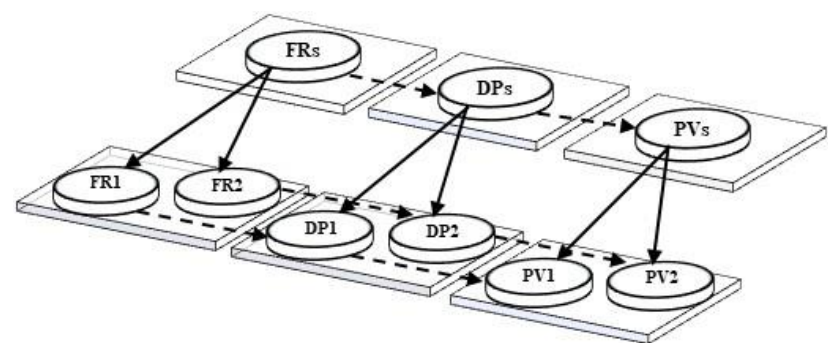

Fig. 5 Representative model of the abstraction level hierarchy

Once the first two steps are complete, the $3^{\text {rd }}$ step involves building the Design Matrix (DM) and represents the link between all functional, design and process domains. For the first two domains, DM must be either diagonal or triangular to ensure a consensus with the independence axiom [20]. As shown in Figure 6, building DM serves as a method of checking and numerically representing consistency between all domains [20]. The $4^{\text {th }}$ step deter- mines whether the DM that has been developed can satisfy the independence axiom, as it aims to manage the communication between all domains.

\begin{tabular}{|c|c|c|c|}
\hline FRs & DPs & DP1 & DP2 \\
\hline FR1 & DP11 & 1 & \\
\hline FR2 & DP2 & & 1 \\
\hline \multicolumn{4}{|l}{} \\
\begin{tabular}{|l|c|c|c|}
\hline FRs & PVs & PV1 & PV2 \\
\hline FR1 & PV1 & 1 & \\
\hline FR2 & PV2 & & 1 \\
\hline
\end{tabular}
\end{tabular}

Fig. 6 Representative model of the developed DM

In the event that the level of consistency required falls short and the defined design has violated the independence axiom, the $2^{\text {nd }}$ step will be re-examined to re-design the identified level for uncoupling the design. However, meeting the requirements of the independent FRs might be unachievable, as highlighted by Frey et al. [22] and Kumar and Tandon [28]. Therefore, the alternative route of incorporating Design Structure Matrix (DSM) method is introduced to overtake and complete the process at this stage, as well as modelling the coupled design. Any move towards using DSM method will be judged by the capability of the design, which has been introduced, to meet the independence axiom.

In either situation, whether satisfying or failing to satisfy the independence axiom, the $5^{\text {th }}$ step in this stage investigates whether FRs have been transformed and captured in the process domain, and whether the related PVs have been allocated. Otherwise, the assembled DM in the $3^{\text {rd }}$ step will be reviewed and re-formed to horizontally decompose FRs into PVs. On the whole, the output from this stage evolves from abstract to detailed concepts as presented in the next stage.

\section{Stage two: Detailed Level Definition}

In this stage, the abstraction level hierarchy is extended and detailed, and this attempts to respond to the question "How does the system work to accomplish the expectations?". Using the decomposition tree and zigzagging of $A D$, the $6^{\text {th }}$ step in this framework decomposes the abstraction level of all constructed functional, design and process domains into the lower levels and interconnects them. This process is led by the primary DPs to define the next level of FRs and the corresponding PVs. In other words, the recognised FRs are correlated to the related DPs and PVs, to conceptualise the design and introduce the next FRs, DPs and PVs. Vertically and horizontally, this step synthesises the abstraction level of DPs in both directions, towards determining the next level elements; as presented in Figure 7. 


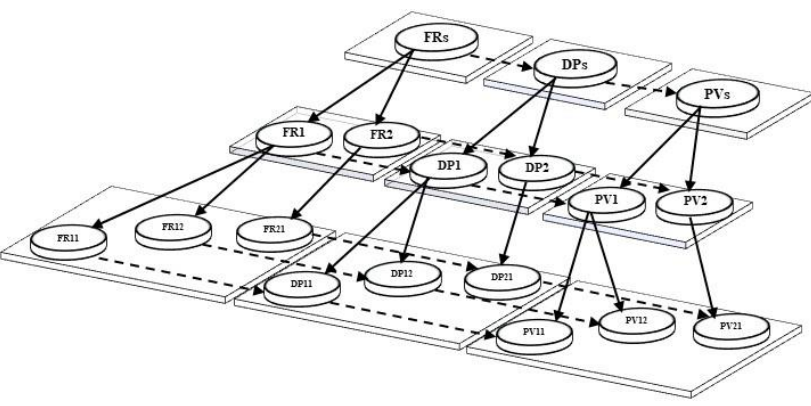

Fig. 7 Representative model of the 2nd level hierarchy

The $7^{\text {th }}, 8^{\text {th }}$ and $9^{\text {th }}$ steps follow the same process as described in the $3^{\text {rd }}, 4^{\text {th }}$ and $5^{\text {th }}$ steps respectively, presented in stage one. Having the abstraction level being decomposed, step 7 builds DM to numerically chase the transformation process and present the whole picture of the integration between the decomposed domains. Next, step 8 assesses the capability of the decomposed domains to ascertain whether the defined system level can fulfil the requirements of the independence axiom. As an alternative, the proposed framework demands two actions at this juncture: either, going back to step 6 and re-decomposing the design elements given towards meeting the condition of this axiom, or establishing DSM as a substitute route to cover and treat the coupling issues between the elements of the decomposed domains. Finally, step 9 evaluates the system level introduced to ensure the decomposed FRs have being captured at the PVs' domain and the associated PVs have been assigned, otherwise step 7 will be evaluated and improved to transform FRs into the PVs' domain. Generally, this stage provides a scoping procedure for executing FMEA and presents the design elements, ensuring they are interconnected in a way that simplifies the process for synchronising Functional, Design and Process FMEAs, which is discussed in the next stage.

\section{Stage three: System Analysis}

This stage involves the process of evaluating the defined hierarchy and seeks to answer the question "How will the defined system proactively improve?". FMEA deployment is brought here to proactively evaluate the design elements, derived from the above stages, and mitigate the associated failures. Step 10 is to apply a detailed FMEA across all the AD domains introduced and to evaluate the potential failure modes associated with all features of the system levels decomposed. Besides, this step determines RPNs; for prioritising and improving the severity and likelihood of failures. Through the constructed hierarchy of all domains, this step addresses the linkage between Functional, Design and Process FMEAs simultaneously in one package.

Having performed FMEA with control actions being determined, steps 11 and 12 in this framework assess the proposed actions and update the DM/DSM respectively: following the FMEA cycle as displayed in Figure 8. The proposed control actions will be traced and evaluated with a new cycle of FMEA to ensure the effectiveness of these actions for reducing the impact or mitigating failures, as well as validating the determined RPNs have been minimised. Once the control actions have been approved, the DM/DSM developed will be updated to demonstrate the design improvements for further investigations that will be covered by the next stage.

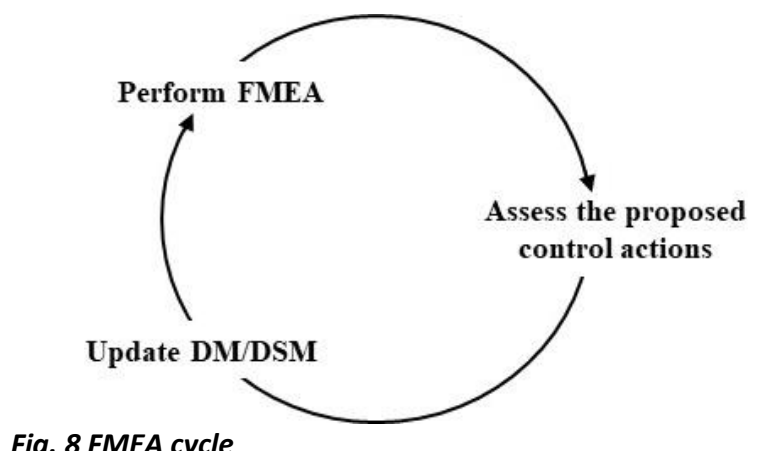

\section{Stage four: Next level Definition}

To continue evolving system analysis, this stage regulates the process of defining the next level of the hierarchy initiated in stage two. It also serves as a determination criterion that either communicates back with stage two to decompose the next level hierarchy of all AD domains, or informs the end of the framework process. This stage is constructed to ensure that the defined hierarchy is achieved and improved by ensuring that all leaf nodes in each hierarchal level are treated. 'Leaf' represents the last item in each tree of all AD hierarchy as presented in Figure 9.

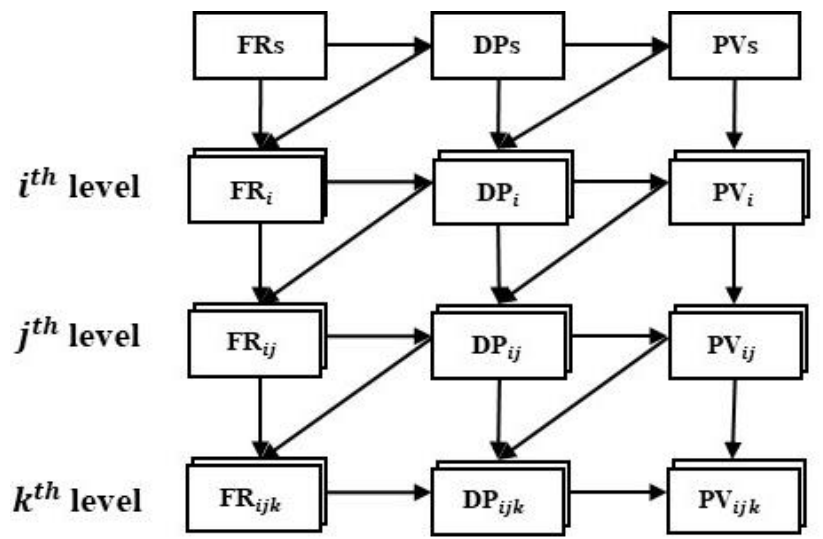

Fig. 9 Representative model of hierarchical leaf

Through the use of expert information, for example engineers' expertise and reference to design manuals, the hierarchy introduced will be appraised to assure that the given design has been understood and proactively investigated. The next steps involve going back to stage two and starting a new iteration to create the next lower level FRs and the associated DPs/PVs. Through the application of FMEA, horizontal correlations between the levels of FRs, DPs and PVs that have been introduced will then be generated and improved. This link and integration are managed by the DM/DSM constructed to explore and represent the correlations between Functional and Design FMEAs over to Process FMEA. At this endpoint, it would help to detect and control the underlying root causes of failures, as this could help to inform that all hierarchical 
leaf nodes are completed. To provide boundaries to the framework's focus and better enable evaluation of its success, this next level definition stage can be limited to the acute elements related to product safety and performance.

\section{Research method for evaluating the developed frame- work}

To practically explore the validity of the proposed framework, a semi-structured questionnaire was formalised to gather experts' feedback and assess the acceptance and performance of the proposed framework by FMEA subject-matter experts. This process seeks to examine the concepts underlying the designed framework, primarily its usefulness, clarity and intuitiveness. Using expert opinions for validation purposes is a common approach and has been used by other works, such as Almutairi, Salonitis and Al-Ashaab [32], Haq and Boddu [33] and Beecham et al. [34].

The questionnaire was made of three parts. The first part records participants' details to assure the right persons are selected. The second part uses a set of closed questions featuring a Likert scale with 5 measures, encouraging participants to assess the framework. Beyond these prior parts, open-ended questions were introduced to capture any extra comments suggested by participants, including the potential limitations in the framework and possible improvements based on the perceptions of the participants. Table 1 presents the assessment criterion and the associated definitions where the questionnaire was built upon.

Table 1

Framework assessment criteria

\begin{tabular}{cll}
\hline Measure & $\begin{array}{c}\text { Assessment } \\
\text { criterion }\end{array}$ & \multicolumn{1}{c}{ Definition } \\
\hline A & Ease of Use & $\begin{array}{l}\text { The degree which implementing } \\
\text { this framework is effortless. }\end{array}$ \\
\hline B & $\begin{array}{l}\text { Useful for imple- } \\
\text { menting FMEA } \\
\text { applications }\end{array}$ & $\begin{array}{l}\text { The degree to which a person be- } \\
\text { lieves that using this framework } \\
\text { would enhance the implementa- } \\
\text { tion of FMEA applications. }\end{array}$ \\
\hline C & $\begin{array}{l}\text { Providing } \\
\text { support }\end{array}$ & $\begin{array}{l}\text { The extent to which the proposed } \\
\text { framework can support synchronis- } \\
\text { ing FMEA applications. }\end{array}$ \\
\hline D & $\begin{array}{l}\text { Comprehensive- } \\
\text { ness }\end{array}$ & $\begin{array}{l}\text { The capability } \\
\text { of how comprehensive the frame- } \\
\text { work is to catch the cascading fail- } \\
\text { ures. }\end{array}$ \\
\hline E & Applicability & $\begin{array}{l}\text { The extent of how likely the user } \\
\text { will be able to use and apply the } \\
\text { framework. }\end{array}$ \\
\hline
\end{tabular}

These assessment criteria are displayed in Likert-scale questions as explained in Table 2.

Table 2

Part two of the questionnaire - the measurement instrument

A. To what extent do you agree that the proposed framework is easy to use?

$\begin{array}{lllll}\square 5 & \begin{array}{l}\square \\ \text { Very easy to use }\end{array} & \begin{array}{l}\square \\ \text { Easy to use with minor } \\ \text { explanations needed }\end{array} & \begin{array}{l}\text { Easy to use but more ex- } \\ \text { planations required }\end{array} & \begin{array}{l}\text { Not easy to use but could be } \\ \text { used with intensive explana- } \\ \text { tions }\end{array}\end{array}$

B. To what extent do you agree that the proposed framework is useful for implementing FMEAs?

\begin{tabular}{lllll}
$\square 5$ & $\square 4$ & $\square 3$ & $\square 2$ & $\square 1$ \\
Very useful & Useful & $\begin{array}{l}\text { Useful but may need } \\
\text { some improvements }\end{array}$ & $\begin{array}{l}\text { Not useful but it could be con- } \\
\text { sidered for use }\end{array}$ & Not very useful \\
\hline
\end{tabular}

C. To what extent do you agree that the proposed framework is providing support with regards to synchronising FMEAs?

$\square 5 \quad \square 4 \quad \square 3 \quad \square 2 \quad \square 1$

Provide a lot of Provide support Provide some support but Does not provide enough sup-

support there would be a need for port but it could be used as an

other tools extra tool

D. To what extent do you agree that the proposed framework is comprehensive, and will catch/detect the cascading failures?
$\square 5$
$\square 4$
Very comprehen- comprehensive
$\square 3$
Fairly comprehensive
$\square 2$
$\square 1$ sive

E. To what extent do you agree that the proposed framework is applicable?

\begin{tabular}{lllll}
$\square 5$ & $\square 4$ & $\square 3$ & $\square 2$ & $\square 1$ \\
More applicable $\quad$ Applicable & $\begin{array}{l}\text { Applicable but requires } \\
\text { some modifications to the } \\
\text { framework }\end{array}$ & $\begin{array}{l}\text { Not applicable unless there will Not applicable at all } \\
\text { framework }\end{array}$ & \\
\hline
\end{tabular}


Every individual measure was formed of 5 scales; ' 0 ' denotes weak performance gradually moving to the excellent measure of ' 5 '.

12 FMEA-related personnel from both the industry and academia with various backgrounds were purposely sampled. Due to the sensitivity of the information being disclosed, the participants and their respective employers are anonymised. Table 3 provides details (i.e. profiles) of the participants involved in the study. To complete the validation process, one-hour meetings were held physically and virtually with participants to collect their feedback and validate the framework. The validation process began with 20 to 30 minutes presentation explained the research, its objectives and the proposed framework. The participants were then asked to assess the framework using the assessment criterion presented in Table 2, giving each question a number/rank from 1 to 5 .

Table 3

Participants profiles

\begin{tabular}{|c|c|c|c|c|}
\hline No & $\begin{array}{l}\text { Participant } \\
\text { role }\end{array}$ & $\begin{array}{l}\text { Participant } \\
\text { expertise }\end{array}$ & $\begin{array}{c}\text { Experience } \\
\text { (Years) }\end{array}$ & $\begin{array}{c}\text { Type } \\
\text { of organisation }\end{array}$ \\
\hline 1 & Consultant & Manufacturing & $>15$ & Design \\
\hline 2 & $\begin{array}{l}\text { Quality } \\
\text { improvement } \\
\text { manager }\end{array}$ & $\begin{array}{l}\text { Manufacturing/ } \\
\text { Quality }\end{array}$ & $>15$ & $\begin{array}{l}\text { Design/ } \\
\text { Manufacturing/ } \\
\text { Consultancy/ } \\
\text { Research centre }\end{array}$ \\
\hline 3 & $\begin{array}{l}\text { Producibility } \\
\text { lead }\end{array}$ & $\begin{array}{l}\text { Design/ } \\
\text { Producibility }\end{array}$ & $11-15$ & Design \\
\hline 4 & Consultant & Software & $>15$ & Software \\
\hline 5 & Consultant & $\begin{array}{l}\text { Quality/ } \\
\text { Metallurgy }\end{array}$ & $>15$ & Consultancy \\
\hline 6 & Researcher & Manufacturing & $<5$ & Research Centre \\
\hline 7 & Researcher & Design & $6-10$ years & Research Centre \\
\hline 8 & Researcher & Manufacturing & $<5$ & Manufacturing \\
\hline 9 & $\begin{array}{l}\text { Senior } \\
\text { Manager }\end{array}$ & Operations & $>15$ & Consultancy \\
\hline 10 & Consultant & $\begin{array}{l}\text { Manufacturing/ } \\
\text { Quality }\end{array}$ & $>15$ & $\begin{array}{l}\text { Design/ } \\
\text { Manufacturing/ } \\
\text { Consultancy/ } \\
\text { Research centre }\end{array}$ \\
\hline 11 & $\begin{array}{l}\text { Sub-system } \\
\text { specialist } \\
\text { Design }\end{array}$ & Design & $>15$ & Design \\
\hline 12 & $\begin{array}{l}\text { integration } \\
\text { engineer }\end{array}$ & Design & $>15$ & Design \\
\hline
\end{tabular}

Inspired by Almutairi, Salonitis and Al-Ashaab [32] and Vinodh and Chintha [35], one sample t-test was implemented to analyse the feedback that was collected from participants. This examined the acceptance level of the proposed framework of synchronising FMEA applications by their perspectives. The t-test is a type of inferential statistical models used to compare means from two different groups, as well as testing the null hypotheses. Equation
(3) shows the basis for the t-test model; estimating t value as follows:

$$
t=\frac{\bar{x}-\mu}{s_{\bar{x}}}
$$

where:

$$
s_{\bar{x}}=\frac{s}{\sqrt{n}}
$$

where:

$\bar{x}$ denotes the sample mean,

$\mu$ typifies the hypothesised mean,

$s_{\bar{x}}$ represents the estimated standard error of the mean.

The symbol $s$ is the sample standard deviation and $n$ is the sample size. In the context of this study, the hypothesised mean was given and assumed by the researcher, for measuring the performance of the proposed framework.

\section{RESULTS}

Led by the gap in the literature, the integrated AD-DSMFMEA framework was designed to incorporate Functional, Design and Process FMEAs into an integrated working architecture and simplify the workflow of FMEA deployment. To assess the acceptance level of the proposed framework, this article investigates different hypothesised mean trials for each evaluation measure in the assessment criterion. The summary of the descriptive statistics is presented in Table 4.

Table 4

\begin{tabular}{|c|c|c|c|c|c|c|c|}
\hline 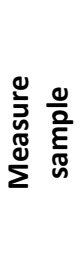 & 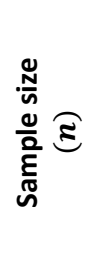 & 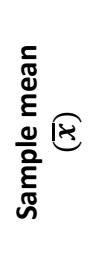 & 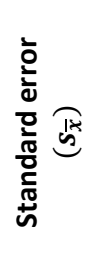 & 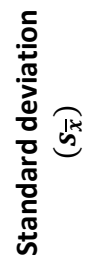 & Min & Max & 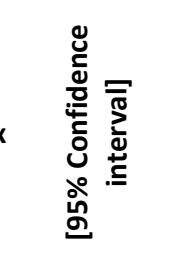 \\
\hline$A$ & 12 & 2.75 & 0.18 & 0.62 & 2 & 4 & $2.355 \quad 3.144$ \\
\hline$B$ & 12 & 4.67 & 0.28 & 0.98 & 2 & 5 & 3.0414 .292 \\
\hline C & 12 & 3.75 & 0.22 & 0.75 & 2 & 5 & 3.2714 .229 \\
\hline$D$ & 12 & 3.58 & 0.23 & 0.79 & 2 & 5 & 3.0794 .087 \\
\hline$E$ & 12 & 3.83 & 0.27 & 0.93 & 2 & 5 & $3.237 \quad 4.429$ \\
\hline
\end{tabular}

The summary of descriptive statistics

For the means of all samples, Table 5 presents the t-test results for the three distinct hypothesised mean trials. The chosen significance level ( $p$-value) for this model is 0.05 . Using this model, the $p$-value of more than $5 \%$ ( $p$-value > 0.05 ) indicates weak evidence against the null hypothesis (HO: $\mu=\bar{x}$ ), so the researchers fails to reject the null hypothesis and the alternative hypothesis $(\mathrm{HO}: \mu \neq \bar{x})$ is being rejected. The $p$-value of less than $5 \%$ ( $p$-value $<0.05)$ indicates strong evidence against the null hypothesis ( $\mathrm{HO}$ : $\mu=\bar{x}$ ), so the null hypothesis is rejected and the alternative hypothesis ( $\mathrm{HO}: \mu \neq \bar{x}$ ) is being accepted.

For the first trial, the hypothesised mean was set to equal 4 , which means that $80 \%$ of the opinions are in favour of the successful application of the proposed framework in practice to achieve the target required for each measure $(A, B, C, D$ and $E)$ at the $95 \%$ confidence interval. On this 
basis, the t-test was specified using STATA (v.16) for all criterion measures at the $95 \%$ confidence intervals. At $\mu=4$, the $p$-values for samples $B, C$ and $E(p=0.13$, 013 and 0.27 respectively) are statistically insignificant and therefore, this model failed to reject the null hypothesises for $B, C$ and $E$ samples while the null hypothesises for $A$ and $D$ samples were rejected.

Table 5

The results of $t$-test model for the three trials

\begin{tabular}{ccc}
$\begin{array}{c}\text { Measure } \\
\text { sample }\end{array}$ & $\begin{array}{c}\boldsymbol{t} \\
\text { value }\end{array}$ & $\begin{array}{c}\boldsymbol{p} \text { - } \\
\text { value }\end{array}$ \\
\hline$A$ & -6.96 & 0.00 \\
$B$ & -1.17 & 0.13 \\
$C$ & -1.15 & 0.13 \\
$D$ & -1.82 & 0.04
\end{tabular}

Trial 1: Reporting that $80 \%$ of the opinions are in favour of the successful implementation of the proposed framework.

$\mathrm{HO}: \mu=4$

Alternative hypothesis

HO: $\mu \neq 4$

Trial 2: Reporting that $70 \%$ of the opinions are in favour of the successful implementation of the proposed framework.

$\begin{array}{ccc}A & -4.18 & 0.00 \\ B & - & - \\ C & - & - \\ D & 0.36 & 0.36\end{array}$

Null hypothesis

$\mathrm{HO}: \mu=3.5$

Alternative hypothesis

HO: $\mu \neq 3.5$

Trial 3: Reporting that $60 \%$ of the opinions are in favour of the successful implementation of the proposed framework.

Null hypothesis

$\mathrm{HO}: \mu=3$

Alternative hypothesis

$\mathrm{HO}: \mu \neq 3$

This demonstrates that the participants believe that the proposed framework is useful, providing support to synchronise FMEAs and practically applicable. Figure 10 presents the participants' responses to each measure scale. A high level of performance (4) was reported for measures $B, C, D$ and $E$, which indicates that most responses point to the framework being practically feasible and adoptable.

Among various trials, the hypothesised mean of the second trial was given as 3.5 to test the other measures; $A$ and $D$. At this trial, the t-test model results suggest only measure $D$ has satisfied the null hypothesis with a $p$-value of 0.36 . This signifies that $70 \%$ of the opinions suggest that the proposed framework is quite comprehensive in terms of its ability to catch and detect failures that could occur and cascade over the product development stages. At the hypothesised mean of 3 , the third trial has confirmed the consensus of measure $A$ with a $p$-value being just above $5 \%$ to null hypothesis of equal 3 . This demonstrates that the $\mathrm{t}$-test model rejected the alternative hypothesis $(\mu=$ 3 ). These findings indicate that the participants believe that this proposed framework would be easy to use if it was supported with further explanations. Overall, the findings have manifested that the proposed framework is accepted and being determined accessible and manageable for some extent with more explanations required, which may be covered by extra applied case studies as suggested by the majority of responses.

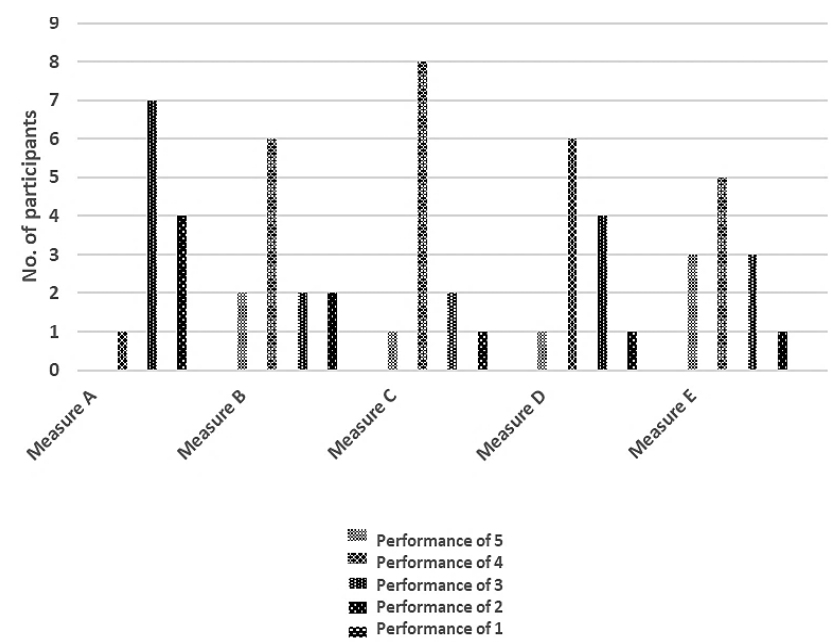

Fig. 10 Participants' responses to all measures

\section{DISCUSSION}

In support of Mohsen and Cekecek's [19] suggestion and extension to Arcidiacono and Campatelli's [23] proposal, this study has developed and validated the proposed framework that co-adopted AD, DSM and FMEA methodologies with the overall aim for synchronising Functional, Design and Process FMEAs. Both the works of Jefferson, Ratchev and Crossley [26] and Guenov and Barker [27] share several key features of this proposed framework. However, the additional feature in this framework is the involvement of FMEA method. This framework might be deemed specifically beneficial for supporting the successful application of $A D$ by evaluating and mitigating the risks underlined and by maximising the reliability of design. However, it is aimed at developing a means for managing FMEAs' synchronicity within complex systems.

The validation findings reveal that the proposed framework could improve the implementation of FMEA and synchronise Functional, Design and Process FMEAs. The transformation processes across the functional, design and process domains could help to correlate the related features through the hierarchal structure developed. Compared to the traditional FMEA form, this framework could decompose the system functions into sub-functions and correlate them to design features, then identify the associated failure modes, causes and control actions. It is considered that a proper decomposition and link between the functional, design and process domains of product development will easily lead to a good quality FMEA interlinkage and reduction of the associated execution cost [1, 3]. 
Indeed, the review of the relevant literature suggests a dearth of research linked to the use of AD method in synchronising all FMEA procedures. However, the results obtained in this study appear to be encouraging in terms of highlighting the effect of AD method to perform this task. The ability of AD method to resolve FMEA synchronisation issues in this regard has also been documented by others $[19,23]$. For example, Arcidiacono and Campatelli [23] developed a systematised model to enhance the application of FMEA for improving the reliability of diesel engine. Besides FTA approach, AD method was mainly incorporated to form Arcidiacono and Campatelli's [23] model. When deployed, this model was found to reduce the time normally taken to undertake FMEA by $50 \%$. As being observed, however, that the authors' model is only limited to Functional and Design FMEAs. Therefore, extending this model to cover the link to Process FMEA could even save more resources as to which can correlate the design failures to their process attributes.

The model results presented in this research suggest that the developed framework of AD-DSM-FMEA could offer such an extension, which in turn could save more resources. This could be seen through the transformation processes of FRs, DPs and PVs across the functional, design and process domains $[3,19,23]$, that feature in the proposed framework. AD approach is believed to provide an effective tool for transmitting and capturing FRs, DPs and PVs throughout the structured trees $[19,20]$. These trees are identified as being more effective for modelling and understanding the effects of failures [11, 23, 36]. From this point, the framework proposed in this research is believed to provide a valuable extension to the proposals suggested by Kmenta and Ishii [4], Cooper [18] and Arcidiacono and Campatelli [23].

Moreover, It should be noted that Arcidiacono and Campatelli's [23] model ignores the fact that there is a coupling that often exists between most modern design elements, and only the application of AD could not assure the required level of synchronisation between Functional and Design FMEAs. This issue was considered by Frey et al. [22] and Kumar and Tandon [28], who argue that the application of AD method in real-life applications is impossible due to the practical concern over the requirement of this method to maintain the independent functional requirements within designs. However, this issue was acknowledged in the proposed framework, whereby DSM method was included in the framework to consider the coupling issues within the design elements. DSM is a wellknown approach for processing connected design elements [26, 27, 29, 30].

However, the validation of the framework developed for integrating Functional, Design and Process FMEAs presents some limitations that may shape the agenda for future investigations. Primarily, the validation process was limited to a subjective evaluation methodology, which may limit the generalisation and utilisation of the proposed framework. Therefore, it is recommended not only to validate the proposed framework on a larger subjective base, but also to apply it to a real working case to facilitate interpretation of the underlying concepts in a more practical, user-friendly form. Moreover, the proposed framework may be constrained for evaluating the reliability of a complex system, that is characterised by an increasing component detail. Still, the proposed framework is theoretically viewed as a means of developing the picture for managing the link between the different applications of FMEA.

\section{CONCLUSION}

The traditional approach of FMEA shows a deficiency in managing the synchronicity between Functional, Design and Process FMEAs and being able to catch the cascading failures. The intent, therefore, behind this work was to search for the requirements to integrate FMEA procedures into a systematic architecture (i.e. an overarching framework) focusing on displaying a series of coherent interlinked FMEAs across product development stages. As a theoretical contribution, a novel AD-DSM-FMEA framework has been developed and qualitatively validated via feedback from 12 field-related individuals from different backgrounds. From participants' perspectives, the findings of this work suggest that this framework is understood and useful for implementing FMEA. However, some further efforts are required to understand the framework more clearly, which can be promoted by a relative practical validation. Besides, the findings obtained indicate that the framework is applicable in practice, able to coordinate the synchronicity process linked to FMEAs implementation and sufficiently comprehensive to catch the cascading failures. On the whole, the validation study implies the feasibility of the proposed framework in practice with a success rate of a minimum of $60 \%$.

However, the practical validation of the framework proposed for integrating FMEA procedures should be considered as a starting point to ensure the greater applicability of this framework which, in turn, would help to enhance its employment in a real-life setting. For more practical adoption, this framework also requires further investigations to illustrate its scalability. This would serve as a major contribution in making this framework more relevant to practitioners in complex applications. One key element which may help in this regard would be to integrate this proposed framework with Model-Based System engineering tools or dedicated FMEA software to deliver the potential benefits of this framework on account of the complexity of the interactions across the domains and system levels, and the consequent volume of data that might be involved.

\section{ACKNOWLEDGEMENT}

The first author would like to thank Shaqra University (Saudi Arabia) for sponsoring his PhD project. 


\section{REFERENCES}

[1] E. Henshall, I. Campean and B. Rutter. "A System Approach to the Development and Use of FMEA in Complex Automotive Applications". SAE International Journal of Materials and Manufacturing, vol. 7(2), pp. 280-290, 2014.

[2] C. Carlson. Effective FMEAs: Achieving safe, reliable, and economical products and processes using failure mode and effects analysis. John Wiley \& Sons, 2012.

[3] S.G. Teng, S.M. Ho, D. Shumar and P.C. Liu. "Implementing FMEA in a collaborative supply chain environment". International Journal of Quality \& Reliability Management, vol. 23(2), pp. 179-196, 2006.

[4] S. Kmenta and S. Ishii. "Scenario-based failure modes and effects analysis using expected cost". Journal of Mechanical Design, vol. 26(6), pp. 1027-1035, 2004.

[5] A. Soufhwee, A. Hambali, M. Rahman and H. Hanizam. "Development of an Integrated FMEA (i-FMEA) Using DAIREC Methodology for Automotive Manufacturing Company". Applied Mechanics and Materials, vol. 315(1), pp. 176-180, 2013.

[6] L.S. Lipol and J. Haq. "Risk analysis method: FMEA/FMECA in the organisations". International Journal of Basic \& Applied Sciences, vol. 11(5), pp. 74-82, 2011.

[7] A. Segismundo and P.A.C. Miguel. "Failure mode and effects analysis (FMEA) in the context of risk management in new product development". International Journal of Quality \& Reliability Management, vol. 25(9), pp. 899-912, 2008.

[8] K.S. Chin, A. Chan and J.B. Yang. "Development of a fuzzy FMEA based product design system". The International Journal of Advanced Manufacturing Technology, vol. 36(1), pp. 633-649, 2008.

[9] D.H. Stamatis. Failure mode and effect analysis: FMEA from theory to execution. Quality Press, 2003.

[10] N. Xiao, H.Z. Huang, Y. Li, L. He and T. Jin. "Multiple failure modes analysis and weighted risk priority number evaluation in FMEA". Engineering Failure Analysis, vol. 18(4), pp. 1162-1170, 2011.

[11] J. Peeters, R. Basten and T. Tinga. "Improving failure analysis efficiency by combining FTA and FMEA in a recursive manner". Reliability Engineering \& System Safety, vol. 172(1), pp. 36-44, 2018.

[12] C. Estorilio and R.K. Posso. "The reduction of irregularities in the use of process FMEA". International Journal of Quality \& Reliability Management, vol. 27 (6), pp. 721-733, 2010.

[13] C. Yang, Y. Zou, P. Lai and N. Jiang. "Data mining-based methods for fault isolation with validated FMEA model ranking". Applied Intelligence, vol. 43(4), pp. 913-923, 2015.

[14] D.M. Ginn, D.V. Jones, H. Rahnejat and M. Zairi. "The $\mathrm{qfd} / \mathrm{fmea}$ interface". European Journal of Innovation Management, vol. 1(1), pp. 7-20, 1998.

[15] Y.K. Gu, Z.X. Cheng and G.Q. Qiu. "An improved FMEA analysis method based on QFD and TOPSIS theory". International Journal on Interactive Design and Manufacturing, vol. 13(2), pp. 617-626, 2019.

[16] F. Shaker, A. Shahin and S. Jahanyan. "Developing a twophase QFD for improving FMEA: an integrative approach". International Journal of Quality \& Reliability Management, vol. 36(8), pp. 1454-1474, 2019.
[17] A. Hassan, A. Siadat, J.Y. Dantan and P. Martin. “Conceptual process planning - an improvement approach using QFD, FMEA and ABC methods". Robotics and Computer-Integrated Manufacturing, vol. 26(4), pp. 392-401, 2010.

[18] H.C. Cooper. "Capture all critical failure modes into FMEA in half the time with a simple decomposition table (Actual case study savings $=\$ 4,206,000)$ ". In 2015 Annual Reliability And Maintainability Symposium (RAMS). 26-29 January, 2015, Palm Harbor, FL, USA, pp. 1-6. ISBN: 978-1-47996703-2.

[19] H. Mohsen and E. Cekecek. "Thoughts on the use of axiomatic designs within the product development process". The First International Conference on Axiomatic Design. 21-23 June, 2000, Cambridge, MA, USA, pp. 188-195.

[20] N. P. Suh. Axiomatic Design: Advances and Applications. Oxford University Press, 2001.

[21] F. Engelhardt. "Improving systems by combining axiomatic design, quality control tools and designed experiments". Research in Engineering Design, vol. 12(4), pp. 204-219, 2000.

[22] D. Frey, J. Palladino, J. Sullivan and M. Atherton. "Part count and design of robust systems". Systems Engineering, vol. 10(3), pp. 203-221, 2007.

[23] G. Arcidiacono and G. Campatelli. "Reliability improvement of a diesel engine using the FMETA approach". Quality and Reliability Engineering International, vol. 20(2), pp. 143-154, 2004

[24] B. Goo, J. Lee, S. Seo, D. Chang and H. Chung. "Design of reliability critical system using axiomatic design with FMECA". International Journal of Naval Architecture and Ocean Engineering, vol. 11(1), pp. 11-21, 2019.

[25] J. Shao, F. Lu, C. Zeng, and M. Xu. "Research progress analysis of reliability design method based on axiomatic design theory". Procedia CIRP, vol. 53, pp. 107-112, 2016.

[26] T.G. Jefferson, S. Ratchev and R. Crossley. "Axiomatic design of a reconfigurable assembly system for primary wing structures". SAE International Journal of Aerospace, vol. 7(2), pp. 229-240, 2014.

[27] M.D. Guenov and S.G. Barker. "Application of axiomatic design and design structure matrix to the decomposition of engineering systems". Systems Engineering, vol. 8(1), pp. 29-40, 2005.

[28] P. Kumar and P. Tandon. "A paradigm for customer-driven product design approach using extended axiomatic design". Journal of Intelligent Manufacturing, vol. 30(2), pp. 589-603, 2019.

[29] S.D. Eppinger and T.R. Browning. Design structure matrix methods and applications. MIT press, 2012.

[30] D. Tang, G. Zhang and S. Dai. "Design as integration of axiomatic design and design structure matrix". Robotics and Computer-Integrated Manufacturing, vol. 25(3), pp. 610619, 2009.

[31] C. Pepe, D. Whitney, E. Henriques, R. Farndon and M. Moss. "Development of a framework for improving engineering processes". Proceedings of the $18^{\text {th }}$ International Conference on Engineering Design (ICED 11) and Impacting Society Through Engineering Design. 15-19 August, 2011, Copenhagen, Denmark, vol. 1, pp. 417-428.

[32] A.M. Almutairi, K. Salonitis and A. Al-Ashaab. "A framework for implementing lean principles in the supply chain management at health-care organizations". International Journal of Lean Six Sigma, vol. 11(3), pp. 463-492, 2019. 
[33] A.N. Haq and V. Boddu. "Analysis of enablers for the implementation of leagile supply chain management using an integrated fuzzy QFD approach". Journal of Intelligent Manufacturing, vol. 28(1), pp. 1-12, 2014.

[34] S. Beecham, T. Hall, C. Britton, M. Cottee and A. Rainer. "Using an expert panel to validate a requirements process improvement model". Journal of Systems and Software, vol. 76(3), pp. 251-275, 2005.

\author{
Mansoor Alruqi \\ University of Nottingham \\ Faculty of Engineering \\ Advanced Manufacturing Building, Jubilee Campus \\ NG8 1BB, Nottingham, United Kingdom
}

Shaqra University, Faculty of Engineering, Saudi Arabia

e-mail: mansoor.alruqi@nottingham.ac.uk

\section{Martin Baumers}

University of Nottingham

Faculty of Engineering

Advanced Manufacturing Building, Jubilee Campus

NG8 1BB, Nottingham, United Kingdom

e-mail:martin.baumers@nottingham.ac.uk

\section{David Branson III}

University of Nottingham

Faculty of Engineering

Advanced Manufacturing Building, Jubilee Campus

NG8 1BB, Nottingham, United Kingdom

e-mail: david.branson@nottingham.ac.uk

\section{Robert Farndon}

Rolls-Royce plc

e-mail: robert.farndon@rolls-royce.com
[35] S. Vinodh and S.K. Chintha. "Leanness assessment using multi-grade fuzzy approach". International Journal of Production Research, vol. 49(2), pp. 431-445, 2011.

[36] R. Ferdous, F. Khan, B. Veitch and P.R. Amyotte. "Methodology for computer aided fuzzy fault tree analysis". Process Safety and Environmental Protection. vol. 87(4), pp. 217226, 2009. 